\title{
Endoscopic ultrasound-guided transesophageal drainage of a mediastinal pancreatic pseudocyst using a novel lumen-apposing metal stent
}

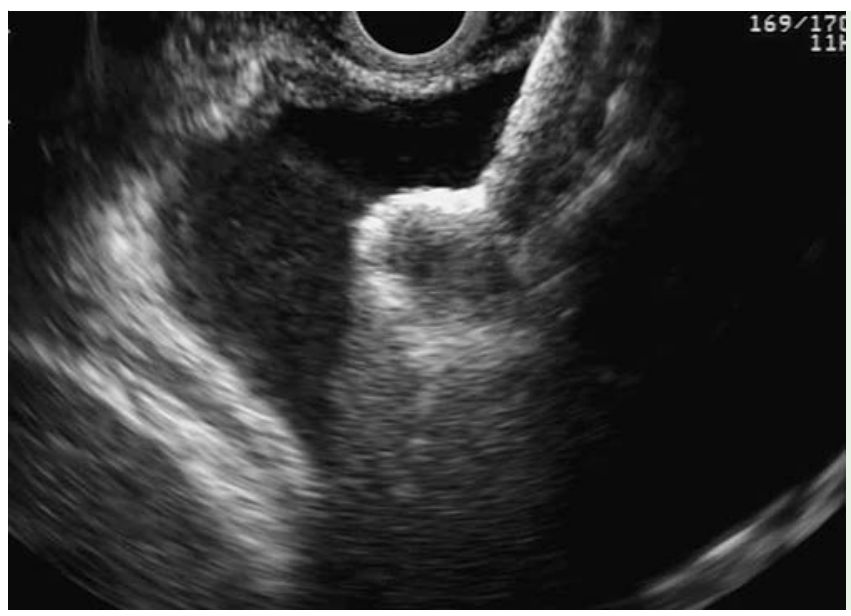

Fig. 1 View during endoscopic ultrasound (EUS)-guided placement of a lumen-apposing metal AXIOS stent across the cystoesophagostomy.

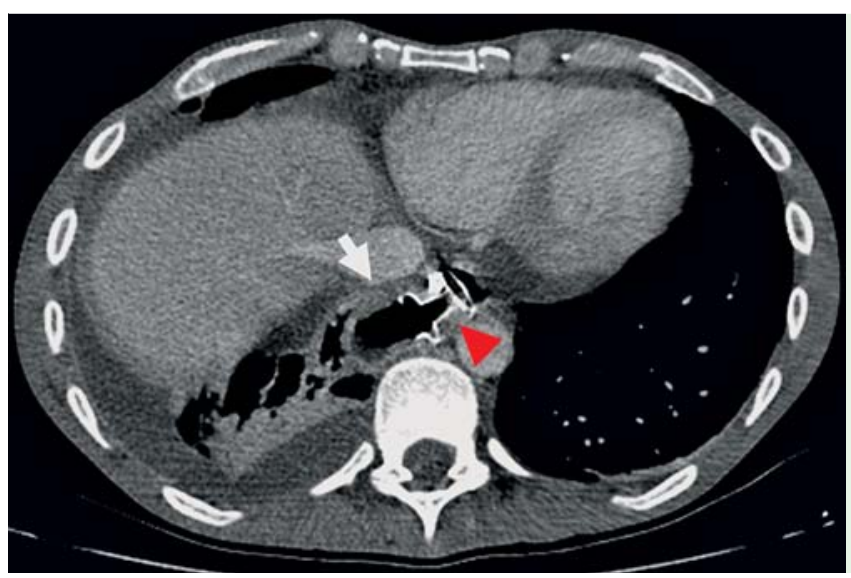

Fig. 3 Follow-up computed tomography (CT) scan after 7 days showing the AXIOS stent (arrowhead) still in place with significant resolution of the lesion (arrow).

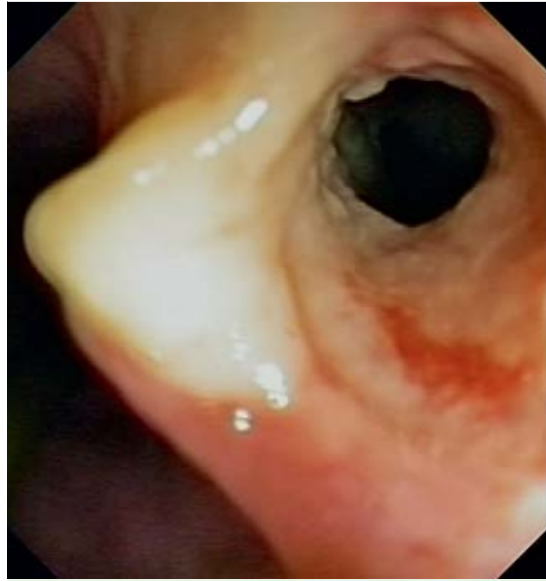

Fig. 4 Endoscopic view of the cystoesophagostomy after the stent had been removed.

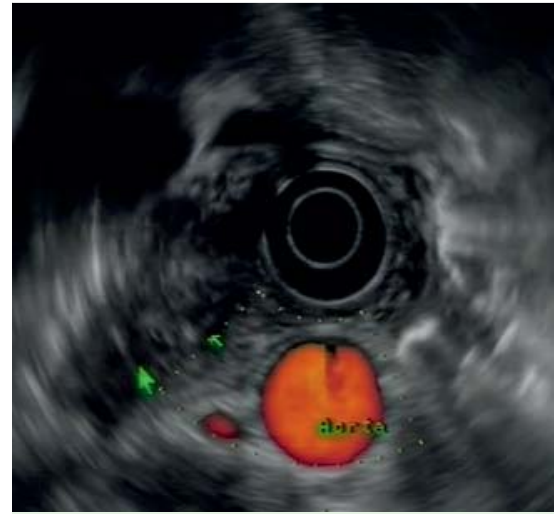

Fig. 5 Follow-up endoscopic ultrasound (EUS) image 6 weeks later showing complete resolution of the lesion.

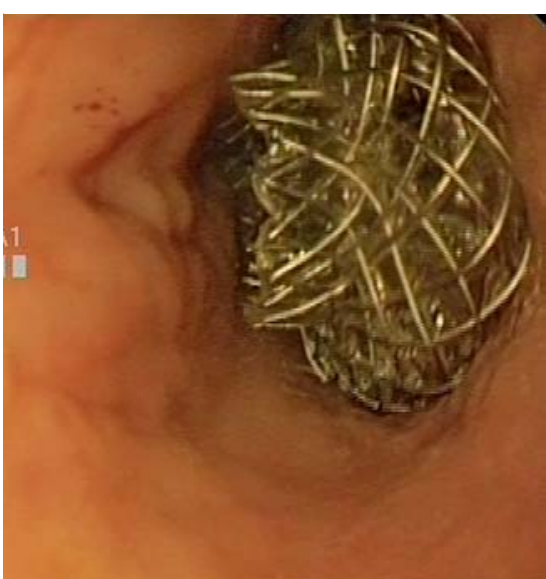

Fig. 2 Endoscopic view of the intraluminal end of the stent within the lower esophagus.

There have been a few previous reports of transesophageal endoscopic ultrasound (EUS)-guided drainage of pancreatic fluid collections (PFC). In these reports the drainage modality has been a single aspiration or deployment of a plastic stent [1-4]. We report a patient who underwent transesophageal EUS-guided drainage of a mediastinal PFC using a novel lumen-apposing metal stent.

A 37-year-old man with a history of rightsided pneumothorax and four episodes of acute pancreatitis was referred for drainage of a PFC. He was experiencing abdominal pain and cysts of increasing size had been seen on his imaging procedures. Computed tomography (CT) scanning revealed an $80 \times 50-\mathrm{mm} \mathrm{PFC}$, which had herniated into the mediastinum adjacent to the lower esophagus.

The PFC was accessed from the lower esophagus using a linear echoendoscope and a novel access device (NAVIX; Xlumena Inc., Mountain View, California, USA) that enables dilation of a tract up to $10 \mathrm{~mm}$ and placement of a guide wire. Once the cystoesophagostomy had been created, a fully covered metal stent with bilateral anchor flanges that can appose nonadherent lumens (AXIOS, 10×10 mm; Xlumena) was placed across the tract $(\diamond$ Fig. 1, $\bullet$ Fig. 2 and $\bullet$ Video 1 ) and 900 $\mathrm{mL}$ of fluid was aspirated. An immediate chest radiograph revealed a tension pneu- 
mothorax on the right side, which required intercostal drainage. The thoracic surgeon who performed the drainage procedure felt that this was a complication of the orotracheal positive pressure.

By day 7 , the patient reported resolution of his abdominal pain and a repeat CT scan revealed a marked reduction in the size of the PFC ( Fig.3). The AXIOS stent was removed ( Fig.4) and the patient was discharged with marked improvement in the pneumothorax. Follow-up imaging after 6 weeks showed complete resolution of the lesion by both EUS and CT scanning ( Fig.5). The patient remains asymptomatic 4 months later.

EUS-guided transesophageal drainage of PFCs has become an alternative to surgery or percutaneous drainage [1-4]. We de-

\section{Video 1}

Transesophageal endoscopic ultrasound (EUS)-guided mediastinal pseudocyst drainage using a lumen-apposing metal AXIOS stent. scribe the first case of transesophageal EUS-guided drainage of a PFC using a novel lumen-apposing metal stent. The procedure was technically successful and led to complete resolution of the lesion, although a pneumothorax occurred as an immediate complication.

Endoscopy_UCTN_Code_TTT_1AS_2AC

Competing interests: None

\section{J. B. Gornals ${ }^{1}$, C. Loras ${ }^{1}$, R. Mast ${ }^{2}$, J. M. Botargues ${ }^{1}$, J. Busquets ${ }^{3}$, J. Castellote ${ }^{1}$}

${ }^{1}$ Department of Digestive Diseases, Hospital Universitari de Bellvitge-IDIBELL, Barcelona, Spain

2 Department of Radiology, Hospital Universitari de Bellvitge-IDIBELL, Barcelona, Spain

${ }^{3}$ Department of Surgery, Hospital Universitari de Bellvitge-IDIBELL, Barcelona, Spain

\section{References}

1 Trevino J, Christein J, Varadarajulu S. EUSguided transesophageal drainage of peripancreatic fluid collections. Gastrointest Endosc 2009; 70: $793-797$

2 Saftouia A, Cuirea T, Dumitrescu D et al. Endoscopic ultrasound-guided transesophageal drainage of a mediastinal pancreatic pseudocyst. Endoscopy 2006; 38: 538-539

3 Baron TH, Wiersema MJ. EUS-guided transesophageal pancreatic pseudocyst drainage. Gastrointest Endosc 2000; 52: 545 - 549

4 Gupta R, Munoz JC, Garg P et al. Mediastinal pancreatic pseudocyst: a case report and review of the literature. Med Gen Med 2007; 9: $8-13$

\section{Bibliography}

DOI http://dx.doi.org/

10.1055/s-0032-1309384

Endoscopy 2012; 44: E211-E212

(c) Georg Thieme Verlag KG

Stuttgart · New York

ISSN 0013-726X

Corresponding author

\section{J. B. Gornals, MD}

Endoscopy Unit

Department of Digestive Diseases

Hospital Universitari de Bellvitge

IDIBELL (Bellvitge Biomedical Research Institute)

Feixa Llarga Str. s/n

08907 L'Hospitalet de Llobregat

Barcelona

Spain

Fax: +34-93-2607681

jgornals@bellvitgehospital.cat 